\title{
CONVEXITY, BOUNDEDNESS, AND ALMOST PERIODICITY FOR DIFFERENTIAL EQUATIONS IN HILBERT SPACE
}

\author{
JEROME A. GOLDSTEIN \\ Department of Mathematics \\ Tulane University \\ New Orleans, Louisiana 70118 U.S.A.
}

(Received October 27, 1978)

ABSTRACT. There are three kinds of results. First we extend and sharpen a convexity inequality of Agmon and Nirenberg for certain differential inequalities in Hilbert space. Next we characterize the bounded solutions of a differential equation in Hilbert space involving and arbitrary unbounded normal operator. Finally, we give a general sufficient condition for a bounded solution of a differential equation in Hilbert space to be almost periodic.

KEY WORDS AND PHRASES. Differential equations in Hilbert space, Convexity inequality, Self-adjoint operators, Bounded solutions, Almost periodic solutions.

AMS (MOS) SUBJECT CLASSIFICATION (1970) CODES. Primary 34605, $47 A 50$ Secondary 34C25, 47BT5. 
1. INTRODUCTION. Let $S_{1}, S_{2}$ be two commuting self-adjoint operators on a complex Hilbert space $H$. Let $u:[a, b] \rightarrow H$ satisfy the inequality

$$
\left\|d u(t) / d t-\left(S_{1}+i S_{2}\right) u(t)\right\| \leq \phi(t)\|u(t)\|, \quad a \leq t \leq b,
$$

where $\int_{a}^{b} \phi(t) d t \leq c<1 / 2$. We shall show that this implies the convexity inequality

$$
\|u(t)\| \leq K_{c}\|u(a)\|^{\frac{b-t}{b-a}}\|u(b)\|^{\frac{t-a}{b-a}},
$$

which holds for some constant $K_{c}$ and all $t \in[a, b]$. S. Agmon and L. Nirenberg [1] first proved this assuming $c=2^{-3 / 2}$; recently S. Zaidman [7] extended it to weak solutions of (1.1). Our results apply to weak solutions and to the range of values $0<c<1 / 2$; moreover, we obtain a smaller constant $K_{c}$ than did these previous authors. This result is presented in Section 2 .

Section 3 is devoted to obtaining the structure of the set of all bounded solutions of

$$
\mathrm{du}(\mathrm{t}) / \mathrm{dt}=\left(\mathrm{S}_{1}+\mathrm{iS}_{2}\right) \mathrm{u}(\mathrm{t}) \quad(-\infty<\mathrm{t}<\infty)
$$

The results generalize and improve a recent result of Zaidman [8].

In Section 4 we study almost periodic solutions of the inhomogeneous equation

$$
d u(t) / d t=A u(t)+f(t) \quad(-\infty<t<\infty) ;
$$

here $A$ is a closed linear operator on $H$ and $f$ is an $H$-valued function. Under a finite dimensionality assumption we show that bounded solutions are almost periodic. This generalizes the results obtained by Zaidman in [6]. 
2. A CONVEXITY THEOREM. Let $u$ map the real interval $[\mathrm{a}, \mathrm{b}]$ into a complex Hilbert space $H$ with inner product $\langle\cdot, \cdot\rangle$. Let $B: D(B) \subset H \rightarrow H$ be a closed, densely defined linear operator. $u$ is a strong solution of

$$
\|d u(t) / d t-B u(t)\| \leq \phi(t)\|u(t)\|
$$

if $u$ is continuously differentiable on $[a, b]$, takes values in $D(B)$, and $f(t) \equiv d u / d t-B u$ satisfies $\|f(t)\| \leq \phi(t)\|u(t)\|, a \leq t \leq b . u$ is a weak solution of (2.1) if $u$ is continuous and for continuously differentiable functions $\psi$ with compact support in $] \mathrm{a}, \mathrm{b}[$ and with values in $D\left(B^{*}\right)$, we have

$$
\begin{aligned}
&-\int_{a}^{b}\left\langle u(t), \psi^{\prime}(t)\right\rangle d t=\int_{a}^{b}\left\{\left\langle u(t), B^{*} \psi(t)\right\rangle+\langle f(t), \psi(t)\rangle\right\} d t, \\
&\|f(t)\| \leq \phi(t)\|u(t)\|, \quad a \leq t \leq b .
\end{aligned}
$$

That a strong solution of (2.1) is a weak solution follows from an integration by parts.

THEOREM 2.1. Let $\mathrm{u}:[\mathrm{a}, \mathrm{b}] \rightarrow H$ be a weak solution of (2.1) where $\mathrm{B}$ is symmetric. If

$$
\int_{a}^{b} \phi(t) d t \leq c<1 / 2,
$$

then the convexity inequality

$$
\|u(t)\| \leq K_{c}\|u(a)\|^{\alpha}\|u(b)\|^{1-\alpha},
$$

holds, where

$$
\alpha=\frac{b-t}{b-a}, \quad K_{c}=\left(\frac{2}{1-2 c}\right)^{1 / 2} .
$$


In particular, when $c=1 / 2 \sqrt{2}$, we get $K_{c}=(4+2 \sqrt{2})^{1 / 2}$. Agmon and Nirenberg [1] proved this result for strong solutions, taking $c=1 / 2 \sqrt{2}$ and obtaining the constant $K_{c}=2 \sqrt{2}\left(>(4+2 \sqrt{2})^{1 / 2}\right)$. This result also appears in Friedman's book [3, p.219]. Zaidman [7] extended the AgmonNirenberg result to weak solutions. The new features of Theorem 2.1 are (i) the result is extended to cover the case $\frac{1}{2 \sqrt{2}}<c<\frac{1}{2}$, (ii) the constant $K_{c}$ is sharpened for each value of $c$ (including $c \leq 1 / 2 \sqrt{2}$ ).

By enlarging the Hilbert space $H$, we can extend $B$ to be a selfadjoint operator (cf. Sz.-Nagy [5]). Also, for $S_{1}$ and $s_{2}$ commuting selfadjoint operators (i.e., $e^{i t S_{1}}$ and $e^{i s S_{2}}$ commute for all real $t$ and $s$ ), we may extend the theorem to the case where $B$ is replaced by the (unbounded) normal operator $S_{1}+i S_{2}$ according to the observation made in $[1$, p.138].

PROOF OF THEOREM 2.1. The proof follows Zaidman [7, pp. 236-244] with the following changes on pp. 242-244. We use Zaidman's notation. From

$$
\|u(t)\|^{2} \leq\left\|u_{1}(b)\right\|^{2}+\left\|u_{2}(a)\right\|^{2}+2 M \int_{a}^{b}\|f(s)\| d s
$$

(cf. $[7$, p.242, line 3]) we get

$$
\|u(t)\|^{2} \leq\left\|u_{1}(b)\right\|^{2}+\left\|u_{1}(a)\right\|^{2}+\varepsilon M^{2}+\varepsilon^{-1}\left(\int_{a}^{b}\|f(s)\| d s\right)^{2}
$$

for each $\varepsilon>0$; here $M=\sup \{\|u(s)\|: a \leq s \leq b\}$. This implies

$$
M^{2} \leq \beta+\varepsilon M^{2}+\varepsilon^{-1} N^{2}
$$

where $\beta=\|\mathrm{u}(\mathrm{a})\|^{2}+\|\mathrm{u}(\mathrm{b})\|^{2}, \quad \mathrm{~N}=\int_{\mathrm{a}}^{\mathrm{b}}\|\mathrm{f}(\mathrm{s})\| \mathrm{ds}$. Consequently

$$
M^{2} \leq\left(\beta+\varepsilon^{-1} N^{2}\right)(1-\varepsilon)^{-1}
$$


for $0<\varepsilon<1$. (This becomes [7, p.242, eqn. (*)] when $\varepsilon=1 / 2$.) Since $u$ is a weak solution of $u^{\prime}-B u=f$ (where $\left.\|f(t)\| \leq \phi(t)\|u(t)\|\right)$, it follows that $\omega_{\sigma}(t) \equiv e^{\sigma t} u(t)$ is a weak solution of $\omega^{\prime}-B_{\sigma} \omega_{\sigma}=e^{\sigma t} f(t)$ where $B_{\sigma}=B-\sigma I$ (cf. [7, Lemma 4, p.242]). Letting

$$
\begin{aligned}
& M_{\sigma}=\sup \left\{\left\|e^{\sigma t} u(t)\right\|^{2}: a \leq t \leq b\right\}, \\
& B_{\sigma}=\left\|e^{\sigma a} u(a)\right\|^{2}+\left\|e^{\sigma b} u(b)\right\|^{2}, \\
& N_{\sigma}=\int_{a}^{b}\left\|e^{\sigma t} f(t)\right\| d t,
\end{aligned}
$$

we have that (2.4) (applied to $\omega_{\sigma}$ rather than $u$ ) yields

$$
M_{\sigma}^{2} \leq\left(\beta_{\sigma}+\varepsilon^{-1} N_{\sigma}^{2}\right)(1-\varepsilon)^{-1}
$$

for all real $\sigma$ and all $\varepsilon, 0<\varepsilon<1$. But by (2.1) and (2.2),

$$
\begin{aligned}
N_{\sigma} & \leq \int_{a}^{b} e^{\sigma t} \phi(t)\|u(t)\| d t \\
& \leq \sup \left\{\left\|e^{\sigma s} u(s)\right\|: a \leq s \leq b\right\} \int_{a}^{b} \phi(t) d t \\
& \leq M_{\sigma} c .
\end{aligned}
$$

Squaring this gives

$$
\mathrm{N}_{\sigma}^{2} \leq \mathrm{M}_{\sigma}^{2} \mathrm{c}^{2} \text {. }
$$

Plugging into (2.5) yields

$$
M_{\sigma}^{2} \leq\left(\beta_{\sigma}+\varepsilon^{-1} c^{2} M_{\sigma}^{2}\right)(1-\varepsilon)^{-1}
$$

or

$$
M_{\sigma}^{2} \leq \frac{\varepsilon \beta_{\sigma}}{\varepsilon(1-\varepsilon)-c^{2}}
$$


provided $0<\varepsilon<1$ and $\varepsilon(1-\varepsilon)>c^{2}$, i.e., $0<c<1 / 2$ and $|2 \varepsilon-1|<\left(1-4 c^{2}\right)^{1 / 2}$. As in [7, pp. 243, 244], u(a) =0 or $u(b)=0$ implies $u \equiv 0$, so to prove the theorem we may suppose $u(a) \neq 0, u(b) \neq 0$. Choosing $\sigma=(b-a)^{-1} \log (\|u(a)\| /\|u(b)\|)$ makes $e^{\sigma t}=(\|u(a)\| /\|u(b)\|)^{\frac{t}{b-a}}$ and $\left\|e^{\sigma a} u(a)\right\|=\left\|e^{\sigma b} u(b)\right\|$. Thus (2.6) becomes, for all $t \in[a, b]$,

$$
\begin{aligned}
\left(\frac{\|u(a)\|}{\|u(b)\|}\right)^{\frac{2 t}{b-a}}\|u(t)\|^{2} & \leq L\left\{\|u(a)\|^{2}\left(\frac{\|u(a)\|}{\|u(b)\|}\right)^{\frac{2 a}{b-a}}+\|u(b)\|^{2}\left(\frac{\|u(a)\|}{\|u(b)\|}\right)^{\frac{2 b}{b-a}}\right\} \\
& =2 L\left(\frac{\|u(a)\|^{2 b}}{\|u(b)\|^{2 a}}\right)^{\frac{1}{b-a}} \cdot
\end{aligned}
$$

where $L=\varepsilon\left(\varepsilon(1-\varepsilon)-c^{2}\right)^{-1}$. Consequently

$$
\|u(t)\| \leq(2 L)^{1 / 2}\|u(a)\|^{\frac{b-t}{b-a}}\|u(b)\|^{\frac{t-a}{b-a}}
$$

holds for $a \leq t \leq b$. Regard $g(\varepsilon) \equiv(2 L)^{1 / 2}=\left(\frac{2 \varepsilon}{\varepsilon(1-\varepsilon)-c^{2}}\right)^{1 / 2}$ as a function of $\varepsilon$. It is minimized when $\varepsilon=c^{\dot{*}}$, in which case $(2 \mathrm{~L})^{1 / 2}=\left(\frac{2}{1-2 \mathrm{c}}\right)^{1 / 2}$. This is a legitimate choice of $\varepsilon$ since $|2 \varepsilon-1|<\left(1-4 c^{2}\right)^{1 / 2}$ holds in this case. The proof of the theorem is now complete.

3. BOUNDED SOLUTIONS. Let $\mathrm{S}_{1}, \mathrm{~S}_{2}$ be commuting self-adjoint operators on $H$. We study functions $u \in C^{1}(\mathbb{R}, H)(\mathbb{R}=]-\infty, \infty[)$ which are bounded (strong) solutions of 


$$
\mathrm{du}(\mathrm{t}) / \mathrm{dt}=\left(\mathrm{S}_{1}+\mathrm{iS_{2 }}\right) \mathrm{u}(\mathrm{t}), \quad t \in \mathbb{R}
$$

LEMMA 3.1. Let $\mathrm{u}$ be a bounded solution of (3.1). Then $\mathrm{u}(\mathrm{t})=\mathrm{e}^{\mathrm{itS}} 2_{\mathrm{h}}$ for all $t \in \mathbb{R}$ and some $h \in \operatorname{Ker}\left(\mathrm{S}_{1}\right)=\left\{f \in H: \mathrm{S}_{1} f=0\right\}$.

PROOF. Let $h=u(0)$. Then

$$
u(t)=e^{t S_{1}}\left(e^{i t S_{2}} h\right)=e^{i t S_{2}}\left(e^{t S_{1}} h\right)
$$

(Recall that $e^{t S_{1}}, e^{i t S_{2}}$ are defined by the operational calculus associated with the spectral theorem.) Since $e^{i t S_{2}}$ is unitary, $\|u(t)\|=\left\|e^{t S_{1}} h\right\|$ follows. But $\left\|e^{t S_{1}} h\right\|$ is bounded for $t \in \underset{\mathbb{R}}{\mathbb{R}}$ if and only if $h \in \operatorname{Ker}\left(S_{1}\right)$, in which case $e^{t S_{1}} h=h$, and so $u(t)=e^{i t S_{2}} h$, as advertised.

A special case occurs when

$$
\operatorname{Ker}\left(\mathrm{S}_{1}\right)=M_{1} \oplus \ldots \oplus M_{n},
$$

where $S_{2}$ restricted to $M_{j}$ is a real constant $\lambda_{j}$ times the identity on $M_{j}$ for $1 \leq j \leq n$. Then any bounded solution of (3.1) is of the form

$$
u(t)=\sum_{j=1}^{n} e^{i t \lambda} j h_{j}
$$

where $h_{j}$ is the orthogonal projection of $u(0)$ onto $M_{j}, 1 \leq j \leq n$. This covers the result obtained by Zaidman in [8]. More precisely, let $\{E(\theta): \theta \in \mathbb{R}\}$ be a resolution of the identity and let

$$
S_{1}=\int_{-\infty}^{\infty} x(\theta) d E(\theta), S_{2}=\int_{-\infty}^{\infty} y(\theta) d E(\theta)
$$

be associated commuting self-adjoint operators, where $x$ and $y$ are continuous 
real functions on $\mathbb{R}$. If the zero set of $x$ is the finite set $\left\{\theta_{1}, \ldots, \theta_{n}\right\}$ then $S_{2}$ is $\lambda_{j}=y\left(\theta_{j}\right)$ times the identity on $M_{j}=\left(E\left(\theta_{j}^{+}\right)-E\left(\theta_{j}{ }^{-}\right)\right)(H)$, $1 \leq j \leq n$, and so any bounded solution of (3.1) is of the form (3.2) with $h_{j} \in M_{j}, 1 \leq j \leq n$. This is Zaidman's result [8].

\section{ALMOST PERIODIC SOLUTIONS.}

THEOREM 4.1. Let $\mathrm{A}: H \rightarrow H$ be a bounded linear operator and let $f: \mathbb{R} \rightarrow H$ be almost periodic. Let $\mathrm{u}: \mathbb{R} \rightarrow H$ be a bounded (i.e. $\sup \{\|u(t)\|: t \in \mathbb{R}\}<\infty)$ strong solution of

$$
d u(t) / d t=A u(t)+f(t) \quad(t \in R) .
$$

Suppose there is a finite dimensional subspace $H_{1}$ of $H$ ) such that $H_{1} \supset\{\operatorname{Af}(s): s \in \mathbb{R}\} \cup\{\mathrm{Au}(0)\}$ and $\mathrm{e}^{\mathrm{tA}}\left(\mathrm{H}_{1}\right) \subset \mathrm{H}_{1}$ for alz $\mathrm{t} \in \mathbb{R}$.

Then $\mathrm{u}$ is almost periodic.

When $H$ is finite dimensional, this is the classical Bohr-NeugebauerBochner theorem (cf. Amerio-Prouse [2, p.85]). When A is a finite rank operator we can take $H_{1}$ to be the range of $A$, and Theorem 4.1 becomes the theorem of Zaidman [6] in this case.

PROOF OF THEOREM 4.1. Let $H_{2}=H \theta H_{1}$ be the orthogonal complement of $H_{1}$, and let $P_{j}$ be the orthogonal projection onto $H_{j}, j=1,2$. Let $u_{j}(t)=P_{j} u(t), j=1,2$. Note that if $L$ is as upper bound for $\|u(s)\|$ $(s \in \mathbf{R})$, then for all real $t$,

$$
\mathrm{L}^{2} \geq\|\mathrm{u}(\mathrm{t})\|^{2}=\left\|\mathrm{u}_{1}(\mathrm{t})\right\|^{2}+\left\|\mathrm{u}_{2}(\mathrm{t})\right\|^{2},
$$

whence $u_{1}$ and $u_{2}$ are bounded. Also, 


$$
\mathrm{du} / \mathrm{dt}=\mathrm{du} \mathrm{u}_{1} / \mathrm{dt}+\mathrm{du} \mathrm{u}_{2} / \mathrm{dt}=A \mathrm{u}_{1}+\mathrm{Au}_{2}+\mathrm{P}_{1} \mathrm{f}+\mathrm{P}_{2} \mathrm{f}
$$

Applying $\mathrm{P}_{1}$ to both sides gives

$$
\mathrm{du}_{1} / \mathrm{dt}=\mathrm{P}_{1} \mathrm{Au}_{1}+\mathrm{P}_{1} \mathrm{Au}_{2}+\mathrm{P}_{1} \mathrm{f}
$$

The function $u$ admits the variation of parameters representation

$$
\begin{aligned}
u(t) & =e^{t A} u(0)+\int_{0}^{t} e^{(t-s) A} f(s) d s \\
& =e^{t A} u(0)+\int_{0}^{t} f(s) d s+\sum_{n=1}^{\infty} \int_{0}^{t} \frac{(t-s)^{n}}{n !} A^{n} f(s) d s .
\end{aligned}
$$

The last (summation) term belongs to $H_{1}$ by (4.2). Applying $P_{2}$ to this expression gives

$$
u_{2}(t)=P_{2} e^{t A} u(0)+\int_{0}^{t} P_{2} f(s) d s ;
$$

differentiating yields

$$
d u_{2}(t) / d t=P_{2} e^{t A} A u(0)+P_{2} f(t)=P_{2} f(t)
$$

by (4.2). Since $f$ is almost periodic and $P_{2}$ is bounded it follows that $\mathrm{du}_{2} / \mathrm{dt}$ is almost periodic. Since $\mathrm{u}_{2}$ is bounded, $u_{2}$ itself is almost periodic (see $[2, \mathrm{p} .55])$.

Next, by (4.3),

$$
d u_{1}(t) / d t=P_{1} A u_{1}(t)+g(t),
$$

where $g(t) \equiv P_{1} A u_{2}(t)+P_{1} f(t)$ is almost periodic. Since $u_{1}$ is bounded and $P_{1} A: H_{1} \rightarrow H_{1}$ is linear, (4.4) is a linear system in the finite 
dimensional Hilbert space $H_{1}$ (see (4.2)). It follows from the classical Bohr-Neugebauer-Bochner theorem [2] that $u_{1}$ is almost periodic. Consequently $u=u_{1}+u_{2}$ is almost periodic, and the proof is complete.

Theorem 4.1 can be easily extended to the case when $A$ is unbounded, as follows.

THEOREM 4.2. Let $\mathrm{A}: \mathrm{D}(\mathrm{A}) \subset \mathrm{H} \rightarrow \mathrm{H}$ generate a $\left(\mathrm{C}_{0}\right)$ group of bounded Zinear operators $\{\mathrm{T}(\mathrm{t}): t \in \mathbb{R}\}$ on $H$ (cf. [4]). Let $u: \mathbb{R} \rightarrow H$ be $a$ bounded solution of (4.1) where $f$ is almost periodic. Suppose there is a finite dimensional subspace $H_{1}$ of $H$ such that

$$
H_{1} \supset\{(T(t)-I) f(s): s \in \mathbb{R}, t \in \mathbb{R}\} \cup\{A u(0)\}
$$

and $T(t)\left(H_{1}\right) \subset H_{1}$ for all $t \in \mathbb{R}$. Then $u$ is almost periodic.

The proof, which differs from the proof of Theorem 4.1 only in inessential ways, is omitted.

COROLLARY 4.3. Let $\lambda_{1}, \ldots, \lambda_{n}$ be eigenvalues of the linear operator $A: D(A) \subset H \rightarrow H$ and let $\phi_{1}, \ldots, \phi_{n}$ be corresponding eigenvectors. Let $H_{1}$ be the span of $\phi_{1}, \ldots, \phi_{\mathrm{n}}$. Then any bounded solution of (4.1) is almost periodic, provided $f: \mathbb{R} \rightarrow H_{1}$ is almost periodic and $u(0) \epsilon H_{1}$.

This follows immediately from Theorem 4.2.

COROLLARY 4.4. In Corolzary 4.3 one can omit the hypothesis that $\mathrm{u}(0) \in H_{1}$ provided that one assumes that $\mathrm{A}$ is a compact normal operator. PROOF. Let $P_{1}, P_{2}, u_{1}, u_{2}$ be as in the proof of Theorem 4.1. Applying $P_{j}$ to (4.1) and noting that $A$ commutes with $P_{j}$ in this case gives 


$$
\begin{aligned}
& d u_{1}(t) / d t=A u_{1}(t)+f(t), \\
& d u_{2}(t) / d t=A u_{2}(t) \quad(t \in \mathbf{R}) .
\end{aligned}
$$

$\mathrm{u}_{1}$ is almost periodic by the Bohr-Neugebauer-Bochner theorem. Thus it only remains to show that $u_{2}$ is almost periodic. Let $B$ be the restriction of $A$ to $\mathrm{H}_{2}$. B is a compact normal operator, hence by the spectral theorem there is an orthonormal basis $\left\{\psi_{\mathrm{m}}\right\}$ for $H_{2}$ and complex numbers $\mu_{\mathrm{m}} \rightarrow 0$ such that

$$
\mathrm{B} \phi=\sum_{\mathrm{m}=1}^{\infty} \mu_{\mathrm{m}}<\phi, \psi_{\mathrm{m}}>\psi_{\mathrm{m}}
$$

for all $\phi \in H_{2}$. Let $Q_{m}$ be the orthogonal projection (in $H_{2}$ ) onto the span of $\psi_{1}, \ldots, \psi_{m}$. Let $v_{m}=Q_{m} u_{2}$. Then

$$
\mathrm{dv}_{\mathrm{m}} / \mathrm{dt}=\mathrm{Q}_{\mathrm{m}} \mathrm{du} / \mathrm{dt}=\mathrm{Q}_{\mathrm{m}} \mathrm{Au_{2 }}=\mathrm{Bv}_{\mathrm{m}}
$$

by (4.5). Also, $v_{m}$ is bounded (since $u_{2}$ is) and takes values in a finite dimensional space, whence $v_{m}$ is almost periodic. We claim that $u_{2}(t)=\lim _{m \rightarrow \infty} v_{m}(t)$, uniformly for $t \in \mathbb{R}$. It then follows that $u_{2}$ is almost periodic [2] and the proof is done. So it only remains to prove the claim. We have

$$
\frac{d}{d t}\left(u_{2}(t)-v_{m}(t)\right)=B\left(u_{2}(t)-v_{m}(t)\right)=\left(B-Q_{m} B\right)\left(u_{2}(t)-v_{m}(t)\right),
$$

therefore

$$
u_{2}(t)-v_{m}(t)=\sum_{k=m+1}^{\infty} e^{t \mu_{k}}<\left(u_{2}-v_{m}\right)(0), \psi_{k}>\psi_{k}
$$

Consequent 1 y

$$
\left\|u_{2}(t)-v_{m}(t)\right\|^{2}=\sum_{k=m+1}^{\infty} e^{t \operatorname{Re} \mu_{k}}\left|<\left(u_{2}-v_{m}\right)(0), \psi_{k}>\right|^{2} .
$$


Since $\left\|u_{2}(t)-v_{m}(t)\right\| \leq\left\|u_{2}(t)\right\| \leq L<\infty$ for some $L$ and all $t \in \mathbb{R}$, it follows that for every $k$ for which $\left\langle\left(u_{2}-v_{m}\right)(0), \psi_{k}\right\rangle \neq 0$ for some $m$, $\mu_{k}$ must be purely imaginary. Therefore

$$
\begin{aligned}
\left\|u_{2}(t)-v_{m}(t)\right\|^{2} & =\sum_{k=m+1}^{\infty}\left|\left\langle\left(u_{2}-v_{m}\right)(0), \psi_{k}\right\rangle\right|^{2} \\
& =\left\|\left(I-Q_{m}\right) u_{2}(0)\right\|^{2} \rightarrow 0
\end{aligned}
$$

as $n \rightarrow \infty$, uniformly for $t \in \mathbb{R}$. Q.E.D.

ACKNOWLEDGEMENT: This research is an outgrowth of discussions I had with Samuel Zaidman in April, 1978 when I had the pleasure to visit the Université de Montréal. I thank Professor Zaidman for some stimulating dicussions, and I gratefully acknowledge that my trip to Montréal was supported by his NRC grant. Finally, I gratefully acknowledge the support of an NSF grant. 


\section{REFERENCES}

[1] Agmon, S. and L. Nirenberg, Properties of solutions of ordinary differential equations in Banach spaces, Comm. Pure Appl. Math. 16 (1963), 121-239.

[2] Amerio, L. and G. Prouse, Almost-Periodic Functions and Functional Equations, Van Nostrand Rheinhold, New York, 1971.

[3] Friedman, A., Partial Differential Equations, Holt, Rinehart and Winston, New York, 1969 .

[4] Hille, E. and R. S. Phillips, Functional Analysis and Semi-Groups, Amer. Math. Soc. Collog. Publ. 31, Providence, R. I., 1957.

[5] Sz.-Nagy, B., Extensions of linear transformations in Hilbert space which extend beyond the space, appendix to F. Riesz and B. Sz.-Nagy, Functional Analysis, Ungar, New York, 1960.

[6] Zaidman, S., Bohr-Neugebauer theorem for operators of finite rank in Hilbert spaces, Atti Acad. Sci. Torino 109 (1974/1975), 183-185.

[7] Zaidman, S., A convexity result for weak differential inequalities, Canad. Math. Bull. 19 (1976), 235-244.

[8] Zaidman, S., Structure of bounded solutions for a class of abstract differential equations, Ann. Univ. Ferrara 22 (1976), 43-47. 


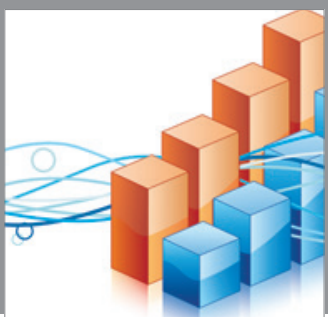

Advances in

Operations Research

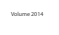

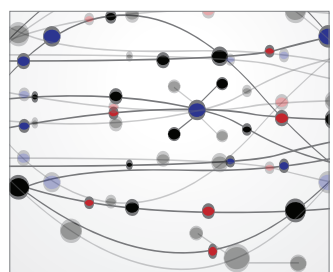

\section{The Scientific} World Journal
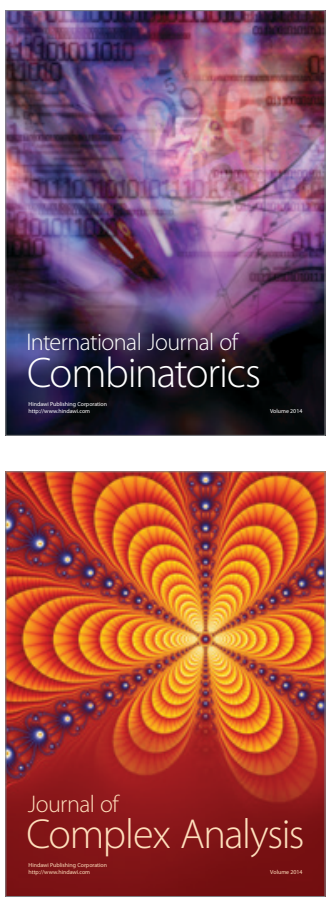

International Journal of

Mathematics and

Mathematical

Sciences
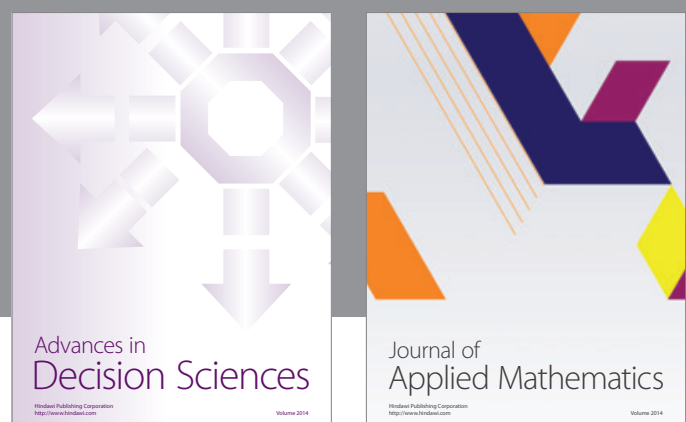

Journal of

Applied Mathematics
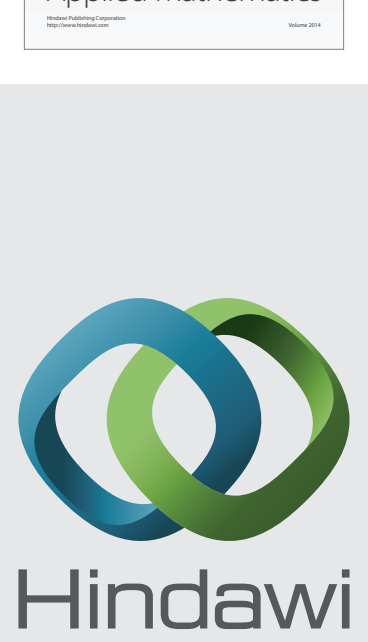

Submit your manuscripts at http://www.hindawi.com
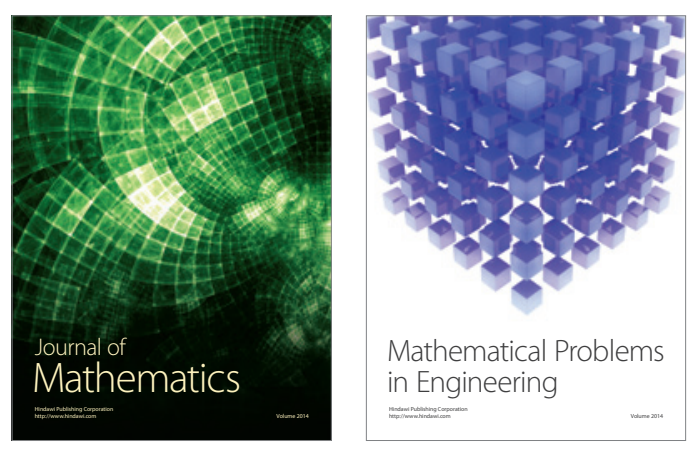

Mathematical Problems in Engineering
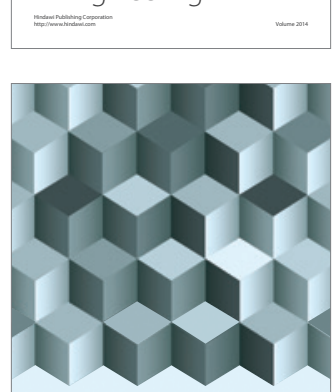

Journal of

Function Spaces
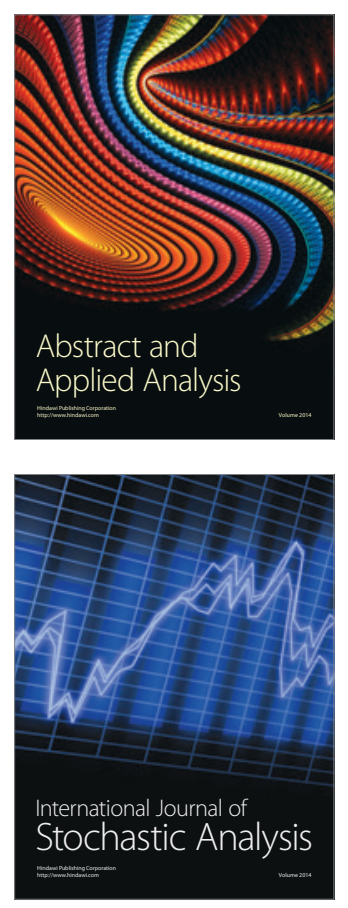

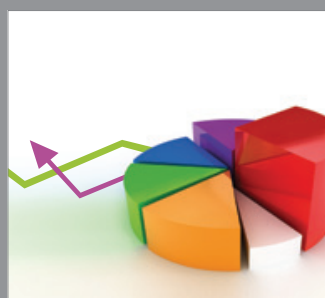

ournal of

Probability and Statistics

Promensencen
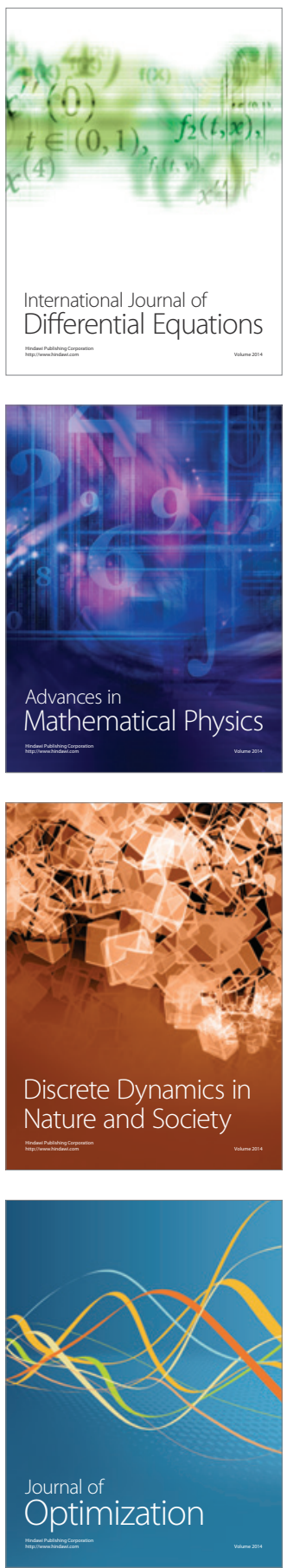